\title{
Testing the Moderating Role of Self-Reliance and Social Support on the Mediated Relationship of Help-Seeking Attitudes and Behavior through Intentions among Individuals with Mental Health Concerns
}

\author{
Mahjalin Araiza Bugtong-Diez \\ University of Batangas, Philippines \\ Gray Matters Psychological and Consultancy Inc.
}

\begin{abstract}
Studies have shown that mental health problems have become a growing public health concern. Despite this, a gap still exists in those who need mental health services and those who seek professional help. Although there are many studies on help-seeking, only a few have focused on thoroughly examining the strength of relationships among the elements of help-seeking, namely, attitudes, intentions, and actual behavior. To gain an improved understanding of what influences professional help-seeking for mental health concerns, the researcher extended the study by adding variables such as self-reliance and social support. The researcher examined if the indirect influence of attitudes toward help-seeking on actual help-seeking behavior through help-seeking intention varies at an individual's different levels of self-reliance and social support on a sample of 604 Filipino participants whose age ranged from 18 to 25 years. A moderated mediation analysis using Hayes' Process Model 9 revealed that none of the investigated factors of help-seeking moderated the relationship between attitudes toward help-seeking and actual help-seeking behavior through help-seeking intention. Nevertheless, the study found that social support predicts actual helpseeking behavior through help-seeking intentions. Thus, an individual who has positive attitudes toward seeking professional help, and who perceives social support for these behaviors will likely seek professional mental help. As such, social support can serve as social encouragement along pathways to mental health care.
\end{abstract}

Keywords: mental health help-seeking attitude, intentions, behavior, self-reliance, social support

\section{Introduction}

One of the common and increasing public health issues in the world is mental health problems. These mental health issues may include concerns ranging from personal difficulties such as adjustment to significant life change to mental illnesses such as depression or anxiety. The World Health Organization (WHO) has recognized psychological health problems as second among the leading causes of disability and impairment in the world (Cairns, Massfeller, \& Deeth, 2010). The prevalence of mental health illness stands as rough evidence concerning the general necessity for mental health treatment in an average population. Studies have shown that while sources of help can be formal or informal, seeking formal help such as psychological services can be more 
beneficial and effective (Rickwood, Deane, Wilson \& Ciarrochi, 2005; National Collaborating Centre for Mental Health, 2004). However, although efficacious therapeutic interventions are available and accessible, formal help-seeking remains marginally an infrequent option for people with psychological problems. This is particularly true with many young adults, who despite having psychological concerns, don't look for help and, subsequently, don't get adequate treatment (Rickwood, Deane, \& Wilson, 2007).

As regards mental health, the most recent definition of this concept denoted it as a coping strategy that reflects an attempt to get external help to manage a mental health issue (Rickwood \& Thomas, 2012). In particular, it means that help-seeking is a process that can vary from the actual behavior of looking for help to intentions to behave to a more general attitude to act in specific ways. This definition sees help-seeking as a behavioral process observing a linear relationship between attitudes, intentions, and behaviors. Furthermore, this definition is also grounded on a particular theory called the theory of planned behavior (TPB) stating that one's attitudes are crucial to the formation of intentions which in consequence affects the actual help-seeking behavior (Azjen, 1991). In this study, the researcher decided on adopting the framework of help-seeking proposed by Rickwood and Thomas in 2012, which narrowed down the focus on attitudes toward help-seeking as the sole predictor of intentions to seek help and excluding the other two, namely, subjective norms and perceived behavioral control. The researcher decided to conceptualize helpseeking using this model to support a more consistent approach of defining and measuring helpseeking.

The current study defined attitudes toward help-seeking as an individual's overall evaluation of obtaining help from professionals in mental health. A few pieces of research revealed that an individual's help-seeking attitudes are amongst the best predictors of treatment-seeking (Carlton \& Deane, 2000; Mackenzie, Gekoski, \& Knox, 2006). It often represents the most considerable variance in help-seeking for problems concerning mental health (Mo \& Mak, 2009; Schomerus, Matschinger, \& Angermeyer, 2009). Evidence also suggests that a reduction in the use of mental health services results from the deepening of one's negative attitudes toward seeking help (Topkaya, 2014). Many studies found support for the connection between attitudes and intentions (Ægisdóttir \& Gerstein, 2009; Choi, 2008). This study described help-seeking intentions as the intent to seek for professional help. An individual's intention is also found to mainly influence actual help-seeking behavior (Armitage \& Conner, 2001).

Whereas help-seeking attitudes and intentions have been viewed as a determinant of helpseeking, self-reliance was found to be a frequently stated reason for not accessing treatment among those having a mental health problem (Zartaloudi, 2010). Thus, dependence on oneself alone serves as a help-seeking barrier that further contributes to the underutilization of mental health services (Chong et al., 2012; Rickwood, Deane, \& Wilson, 2007; Salaheddin \& Mason, 2016). Another crucial factor is social support which denotes free and accessible assistance that comes from one's social network (Rickwood et. al., 2005). Based on some studies, high levels of social support were associated with more negative professional help-seeking attitudes and lower intentions to obtain mental health services (Vogel, Wester, Wei \& Boysen, 2005; Powell \& Kotschessa, 1995).

\section{Research Purposes/Significance}

A few studies uncovered that even though a relationship exists between help-seeking attitudes and intentions, enhancing attitudes does not automatically result in increased help-seeking intentions (Ægisdóttir, O'Heron, Hartong, Haynes, \& Linville, 2011; Christopher, Skillman, Kirkhart, \& D'Souza, 2006). Thus, examining variables that potentially affect the relationship between the two is essential. The researcher believed that extending the current research via the addition of variables 
that have antecedently been considered necessary to influence seeking help (self-reliance and social support), may provide a more complete or better theoretical understanding of this construct. Combining these two moderators, self-reliance and social support will help give a more complete picture of help-seeking as it gives light to the internal and external factors affecting one's intention to seek psychological professional help.

The findings of the research will be necessary to society considering the prevalence of untreated mental health concerns and disorders. Many individuals do not seek help despite knowing that it may be necessary for them because of some factors or barriers that prevent them from doing so. The higher demand for preventing this rate of untreated mental illnesses to increase justifies the need to fully explore the interaction of variables known to influence help-seeking for psychological or emotional problems. The researcher has confidence in the significance of seeing completely the relationship among the variables of help-seeking first before making more powerful interventions in increasing help-seeking behavior. The researcher thought that the implications of the results can assist in modifying or improving mental health help-seeking interventions.

\section{Research Objective and Hypothesis}

Thus, in this study, the researcher endeavored to test the association among help-seeking attitudes, help-seeking intention, actual help-seeking behavior, self-reliance, and social support. Specifically, the researcher intended to analyze the moderating effects of self-reliance and social support in the mediating role of help-seeking intention in the relationship between attitudes toward help-seeking and actual help-seeking behavior. The researcher aimed to test if the indirect influence through help-seeking intention varies at an individual's different levels of self-reliance and social support. For this research problem, the researcher hypothesized that the influence of attitudes toward helpseeking on actual help-seeking behavior through help-seeking intentions is moderated by selfreliance and social support of individuals with mental health concerns, specifically, the influence through help-seeking intentions will be stronger among people with low self-reliance and low social support.

\section{Literature Review}

\section{Attitudes toward help-seeking and Actual help-seeking behavior}

There is evidence showing that attitude consists of the expectations or beliefs an individual has about the dangers and advantages of seeking help (Vogel \& Wester, 2003). For instance, if one is certain that help-seeking from a mental health professional will result in to change in symptoms, feelings, or behavior, and one assesses this change as favorable, at that point one is said to demonstrate an optimistic attitude to obtain help (Ajzen \& Fishbein, 2000). Evidence also suggests that attitudes towards help-seeking for concerns relating to mental well-being significantly correlate with service utilization, such that reduction in the use of mental health services results from the deepening of one's negative attitudes toward seeking help (ten Have et al., 2010; Topkaya, 2014).

\section{Attitudes toward help-seeking and Help-seeking intentions}

While there are several studies on attitudes' association with actual help-seeking behavior, there are also many studies that found support for the connection between attitudes and intentions (Ægisdóttir \& Gerstein, 2009; Choi, 2008; Segal, Coolidge, Mincic, \& O’Riley, 2005; Vogel et al., 2005). People who held optimistic beliefs about the adequacy of psychological treatment services were probably going to show an intent to get treated. Although many studies support this argument and have likewise shown that it bears impacts on help-seeking intentions (Bagley, 2011; Harewood, 2009), there are; however, some studies showing attitudes' weak correlation with intentions to seek help. For instance, one study demonstrated that improving one's attitudes toward help-seeking is possible even without significantly impacting help-seeking intentions, suggesting the idea that 
variables influencing help-seeking intentions may not be limited to attitudes (Christopher et al., 2006).

\section{Help-seeking intentions and Actual help-seeking behavior}

Behavioral intention is interpreted as one's motive to take a particular action, (Ajzen \& Fishbein, 2000 ) or in this case, is the person's intent to engage in help-seeking. Reavley, Yap, Wright, and Jorm's (2011) findings stated that help-seeking intentions are more potent than views about the effectiveness of interventions in promoting help-seeking behavior. Nevertheless, there are also some studies showing the inconsistent relations between intentions and actual behavior regarding help-seeking. Based on Yusuke (2017) \& Nagai (2015), this relationship is not necessarily high inferring that measurement of help-seeking intentions cannot substitute for the analysis of actual help-seeking behavior.

\section{Self-reliance and Social support as possible barriers to seeking professional help}

A study suggests that increased reliance on oneself does not encourage the actual behavior of obtaining help in young individuals with depression and suicidal tendencies (Labouliere, Kleinman, \& Gould, 2015). Roughly thirty-three percent of discouraged and self-destructive youth reflects a higher preference to solve their mental health concerns alone. Besides, adolescent's inclinations to rely on self amid tough circumstances and experiences of mental health difficulties extend to a decision to self-help (Gulliver, Griffiths, \& Christensen, 2010).

In addition to increased self-reliance, people who obtain a high level of social support from individuals in their interpersonal circle probably won't consider seeking professional help even when they encounter mental health issues. Although social support is a resource for coping and is important for adjustment, having a high perceived social support from family and friends could discourage planning to seek professional psychological help (Turner \& Quinn, 1999; Lukito Setiawan, 2006). Martinaez-Hernaez, DiGiacomo, Carceller-Maicas, Correa-Urquiza \& MartorellPoveda (2014) found in their study that the primary cause for avoiding professional services is a reliance on alternative resources such as the social network.

\section{Theoretical Framework}

The conceptual framework used in this study is in line with the theory of approach-avoidance conflict. This theory presumes that conflict occurs when there is a behavior or goal that has both motivational influences and inhibitory influences of differing strengths which thus make the goal pleasant and unpleasant simultaneously (Elliot \& Covington, 2001). The researcher used this theory in conceptualizing help-seeking as being an approach or avoidance conflict where a positive help-seeking attitude is an approaching factor that strengthens the possibility that one will seek out services while an increased level of self-reliance and social support are avoidance factors that weaken this possibility. 


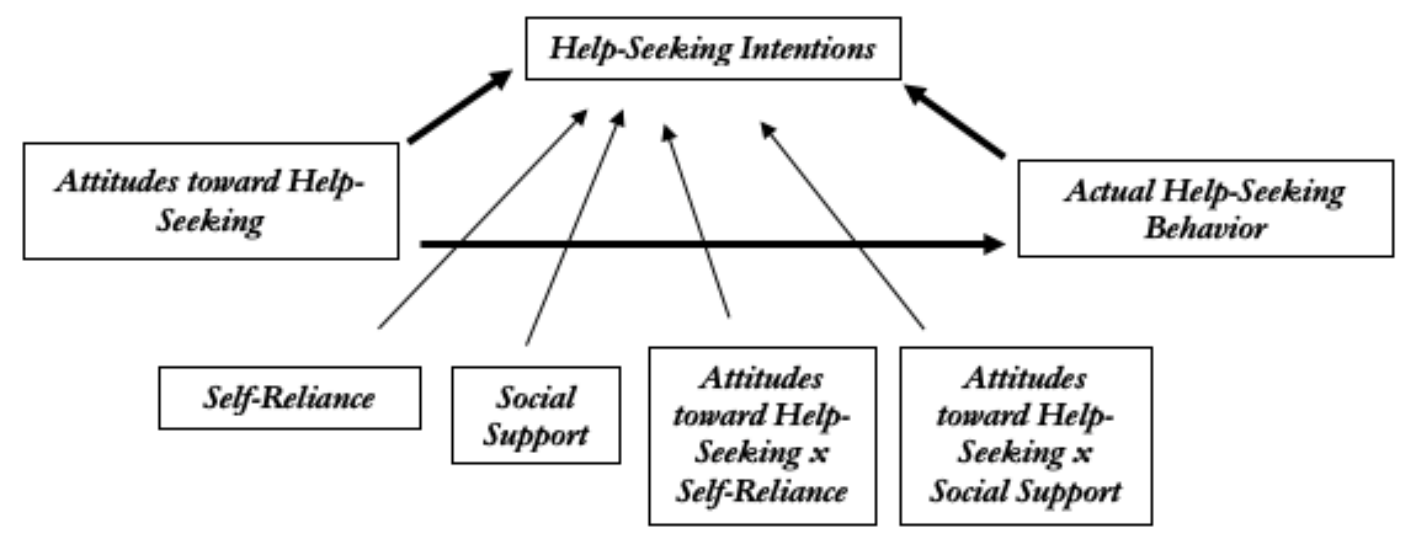

Figure 1: Framework showing that Self-reliance and Social Support moderate the Relationship between Attitudes toward help-seeking and Actual help-seeking behavior through Help-seeking intentions

\section{Method}

\section{Participants and Design}

The researcher employed a quantitative research design. Specifically, it was a survey method that involved the administration of a questionnaire to obtain data on help-seeking attitudes, intentions, behaviors, and self-reliance and social support of individuals with mental health concerns. Klandermans (2013) suggested that research using questionnaires enables one to collect and analyze data from only a few people considered to be characteristic of the targeted entire population while guaranteeing confidentiality and providing information in a relatively brief time at a low cost to the researcher. Data were gathered from a sample of Filipino emerging adults $(\mathrm{N}$ $=604)$ aged 18 to $25(\mathrm{M}=21, \mathrm{SD}=2.40)$. Of them, $50 \%$ were males $(\mathrm{n}=302, \mathrm{M}=20.57, \mathrm{SD}=$ 2.48 ) and $50 \%$ were females ( $\mathrm{n}=302, \mathrm{M}=21.69, \mathrm{SD}=2.19)$, ensuring that both men and women were equally represented in the study. Based on the conclusions of Bujang, Sa'at, and Tidik (2017), a minimum sample size of 300 for non-experimental studies is needed to produce closer estimates with the parameters in the population.

Sampling was carried out using snowball sampling by asking participants to suggest another person who might be suited for this research. Additional efforts to recruit participants were made by tapping institutional resources. This non-probability sampling method was used as it was difficult for the researcher to access the participants with the target characteristics. The participants with ages ranging from 18-25 years old were the focus of the study as this age range implies a higher risk for developing mental health illness yet lower rates of mental health services use (Grant \& Potenza, 2010; Perlick, Hofstein, \& Michael, 2010; Tanner, 2014). Also, for the participants to take part in the study, they should have experienced troubling problems or concerns within the past six months which then subjected them to seek or not seek professional help. Studies show that being in a distressing situation prompts one to ask for help (Jordanova et al., 2007; Rubio \& Lubin, 1986; Solomon, Mikulincer, \& Flum, 1989). More so, the reason for limiting the study on the experience of a troubling problem within the past six months is that stressors that occurred before six months may have already been resolved or the person may have already adjusted to that experience in his or her life. On the other hand, for someone who just had a recent problem, the experience may still be fresh in his or her memory, and the sorrow can be intense, thereby needing more resources to cope with the pain he or she is suffering. Also, Brown \& Harris (1989) also found that six months is the most critical period for predicting distress. Likewise, there are mental 
health conditions such as adjustment disorders where symptoms often dissipate within six months (American Psychiatric Association, 2013).

\section{Materials}

Negative Life Events Scale for Students (NLESS; Buri, 2018) is a checklist intended for use as a screening tool to identify individuals that would fit the population of interest for the current study to be conducted. Mental Help-Seeking Attitudes Scale (MHAS; Hammer, Parent, \& Spiker, 2018) was used as a measure of the study's predictor variable, attitudes toward help-seeking. Mental Help Seeking Intention Scale (MHSIS; Hammer \& Spiker, 2018) was used to measure the mediator variable help-seeking intentions. And just like how it was done in earlier studies, actual help-seeking behavior was measured using this question, "Within the past six months, have you obtained help from a professional for mental health concerns which include but are not limited to problems coping with distressing situations or life events, anxiety, and depression?" (Eisenberg, Golberstein, \& Gollust, 2007; Franz, 2012; Zivin, Eisenberg, Gollust, \& Golberstein, 2009). Participants were considered as having an actual help-seeking behavior if they have obtained professional help within the past six months after finding themselves being in a distressing situation. Self-Reliance for coping with mental health problems (Self-Reliance - MHP; Britt et al., 2011) is a three-item questionnaire that was used to measure moderator variable self-reliance. Multidimensional Scale of Perceived Social Support (MSPSS; Zimet, Dahlem, Zimet, \& Farley, 1988) is a scale consisting of 12 items that measure the other moderator variable, social support.

\section{Procedure}

This study had two parts. First, there was a screening of qualified participants using NLESS Only those participants who reported having at least one troubling concern within the past six months continued to the next part of the study. Second, the participants answered an additional set of questionnaires. Following the NLESS, five sets of questionnaires including MHSAS, MHSIS, Actual Help-Seeking Behavior, Self-Reliance - MHP, and MSPSS were then given. The researcher ensured to always counterbalance the order of the questionnaires to guard against order and sequence effect. Finally, the researcher debriefed the participants about the study and expressed her gratitude for participating. A leaflet about help-seeking for mental health problems was provided right after they answered the questionnaire set. This leaflet contains necessary information about mental health problems as well as contact details of psychologists and clinics that offer mental health services. Likewise, on the online version of the questionnaire, a Google link containing information about mental health clinics/professionals was included. Ethical approval was sought and obtained from the relevant authorities and panelists at the De La Salle University.

\section{Data Analysis}

The data were carefully screened before conducting statistical analyses to guarantee the accuracy of the data file. There have been no missing data as all participants completed all items on every scale. Bootstrapping was observed to deal with the outliers. Before data analysis, items in each instrument were factor analyzed ensuring that constructs are unidimensional. Each item in the instruments used namely MHAS, MHSIS, Self-Reliance - MHP, and MSPSS did not fail to load on a single factor solution. Then, a reliability analysis was completed for each instrument via the omega coefficient. For all the instruments mentioned above, no items that attenuated the value of the omega coefficient were identified. After these properties were established, the researcher proceeded to descriptive analysis and testing of its hypothesis. The researcher made use of model 9 of the PROCESS macro developed by Hayes (2015) to gain an insight into the moderated mediation model. Multiple regression analyses were conducted to weigh each component of the proposed moderation mediation model. A 10,000 bias-corrected bootstrap sample and a 95- 
percentile confidence interval estimate were requested in the PROCESS. Also, to achieve the main objective of the study, binary logistic regression was performed on the independent variable.

Results

Descriptive Statistics

Table 1. Means and Standard Deviations

\begin{tabular}{lclccc}
\hline Variable & N & Mean & SD & Min & Max \\
\hline Help-Seeking Attitude. & 604 & 5.691 & $\mathbf{0 . 9 4 4}$ & 1.778 & 7.000 \\
Self-Reliance & 604 & 2.940 & 0.824 & 1.000 & 5.000 \\
Social Support & 604 & 5.425 & 1.124 & 1.000 & 7.000 \\
Help-Seeking Intention & 604 & 5.107 & 1.364 & 1.000 & 7.000 \\
Actual Help-seeking Behavior & 604 & 0.1126 & 0.3613 & 0.000 & 1.000 \\
\hline
\end{tabular}

Typical ratings on help-seeking attitudes items went from 1.78 to 7.00 with a mean of $5.70(S D=$ 0.94). The respondents' levels of self-reliance ranged from 1 to 5 with a mean of $2.90(S D=0.82)$. Vis-à-vis the level of social support, the analysis showed a mean of $5.43(S D=1.12)$. The respondents' help-seeking intentions ranged from 1.00 to 7.00 with a mean of $5.12(S D=1.36)$ and their actual help-seeking behavior ranged from 0 to 1 with a mean of $0.11(S D=0.32)$. Refer to Table 1.

\section{Moderated Mediation Analysis}

The data was analyzed through Hayes' Process analysis for first stage dual moderated mediation following the Model 9 path.

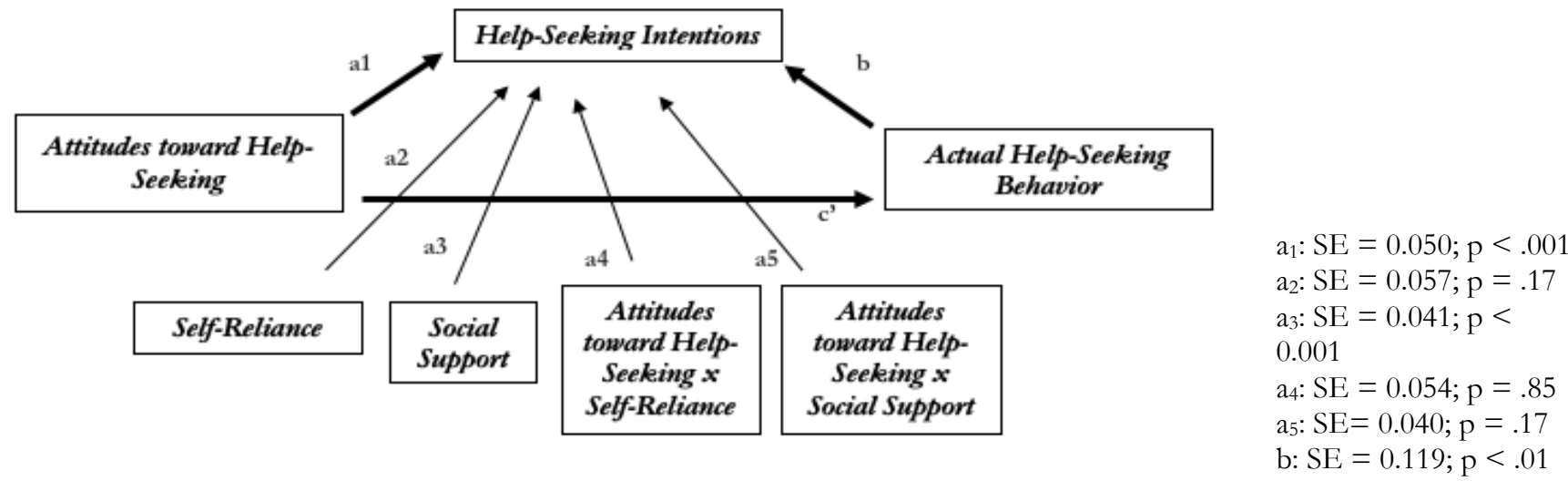

Figure 2: The Moderating Roles of Self-Reliance and Social Support on the relationship between attitudes toward help-seeking and actual help-seeking behavior through help-seeking intentions 
Percentile bootstrap CI based on 10,000 bootstrap samples.

${ }^{*} \mathrm{p}<.05 .{ }^{* *} \mathrm{p}<.01 . * * * \mathrm{p}<.001$.

Table 2: Ordinary least squares regression coefficients (with standard errors) from a first stage dual moderated mediation model

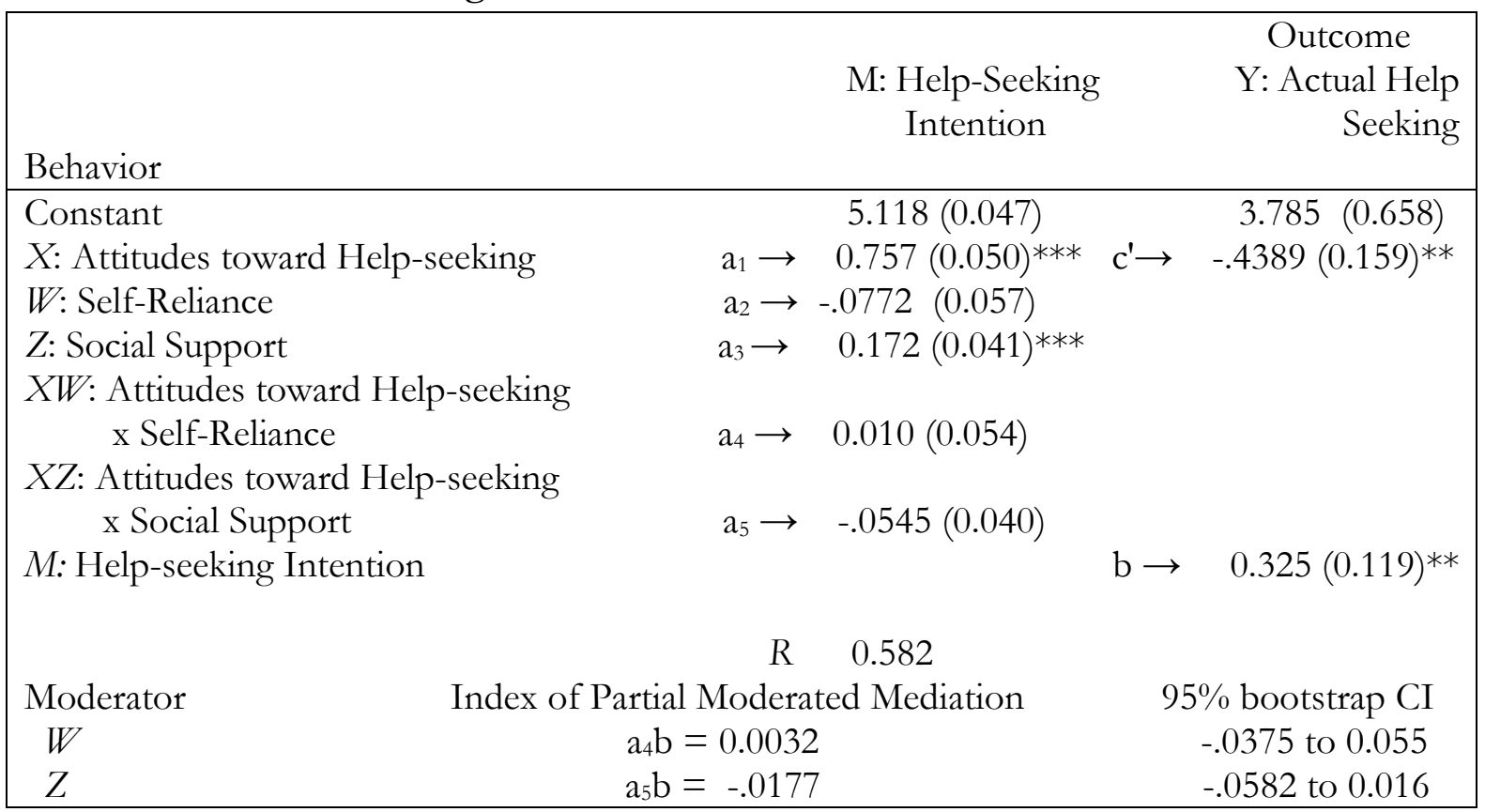

In the model of help-seeking intention $(\mathrm{M})$, self-reliance $(\mathrm{W})$ did not significantly moderate the indirect effect of attitudes toward help-seeking on actual help-seeking behavior $(a 4=0.010, p$ $=.853,95 \% C I=-.095$ to 0.115$)$, as well as social support $(\mathrm{Z})(a 5=-0.0545, p=.173,95 \% C I=$ -0.133 to 0.024$)$. Because the model shows that both the interactions are non-significant, it is, therefore, unnecessary to discuss the conditional indirect effects of attitudes toward help-seeking and actual help-seeking behavior through help-seeking at different values of self-reliance and social support. Also, while no interactions can be observed, an additive effect can be seen in the relationship among attitudes toward help-seeking, social support, and actual help-seeking behavior.

In testing moderated mediation, Hayes (2015) argued that it is more important to consider indices of partial moderated mediation, that is, the weights for self-reliance (W) and social support $(Z)$ in this equation: $a 4 b=0.00325$ and $a 5 b=-0.0177$, respectively. Inference about partial moderated mediation comes from a boot-strap CI for these indices, which when estimated using 10,000 bootstrap samples (see Table 2) is -.0375 to 0.055 for self-reliance (W) and -.0582 to 0.016 for social support $(Z)$. As the $C I$ for the index of partial moderated mediation by self-reliance includes zero, it can be concluded that independent of any moderation of the indirect effect of attitudes toward help-seeking by social support, self-reliance does not moderate this indirect effect. Put simply, even when social support is held fixed, the indirect effect is unrelated to the selfreliance. Likewise, as the $\mathrm{CI}$ for the index of partial moderated mediation by social support includes zero, it can be said that independent of the effect of self-reliance on the indirect effect, the evidence does not definitively support a claim that the indirect effect differs between those with high social support and those with low social support.

The results also revealed that only the moderator, social support, significantly predicts help-seeking intention $(b=0.172, p<.01,95 \% C I=0.092$ to 0.252$)$, indicating a mediational role of help-seeking intention on the relationship between social support and actual help-seeking 
behavior. A post hoc analysis specifically mediation analysis was conducted to confirm the findings that social support predicts actual help-seeking behavior through help-seeking intentions.

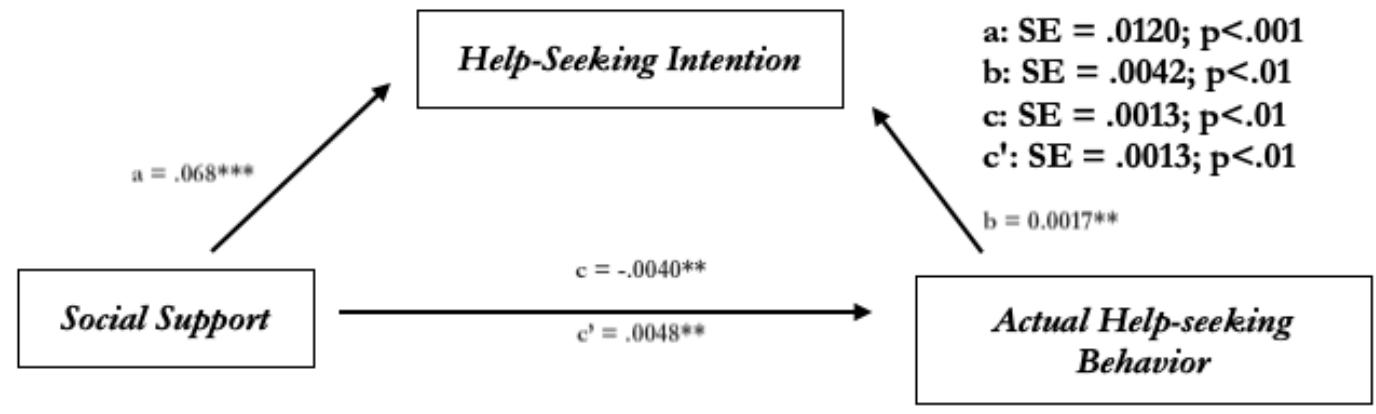

Figure 3: Mediational Role of Help-Seeking Intention on the Relationship Between Social Support and Actual Help-Seeking Behavior

The said post hoc analysis followed Model 4 (Refer to Figure 3), which suits the simple mediation model. The analysis only involved the previous moderator social support as the independent variable and help-seeking intention as a mediator to check if there was a significant change in the dependent variable, actual help-seeking behavior if attitudes toward help-seeking, and self-reliance was not present in the analysis. To conduct this analysis, three paths were observed (Refer to Table $3)$.

Table 3: Post Hoc Analysis of the Relationship of Social Support and Actual HelpSeeking Behavior through Help-Seeking Intention

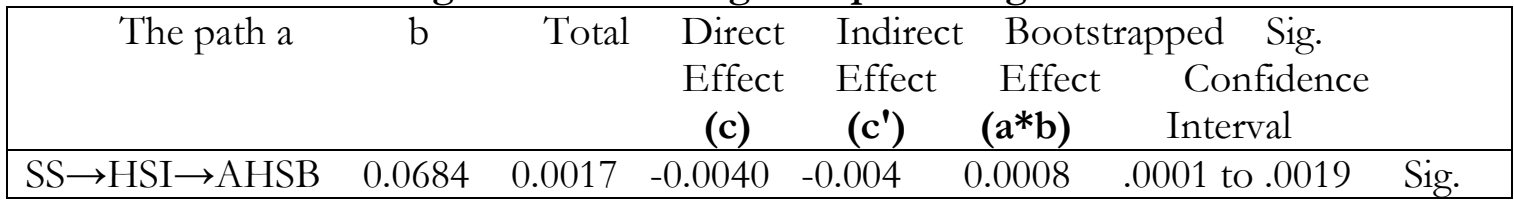

\section{Discussion}

The results of the study can be summarized into two points: firstly, social support, and self-reliance do not moderate the indirect effect of attitudes toward help-seeking on actual help-seeking behavior through help-seeking intentions. Secondly, only social support predicts actual helpseeking behavior through help-seeking intentions, and besides, it has an additive effect on the relationship between help-seeking attitudes, intentions, and behavior. In explaining the results, the researcher looked into the relevance of cultural influences in the samples.

Social Support and Self-Reliance do not moderate the indirect effect of Help-Seeking Attitudes on Actual Help-Seeking Behavior through Help-Seeking Intention

Culture holds an influential role in the formation and development of character and attitudes of people, including individuals with mental health issues (Abdulla \& Brown, 2011). When people have a poor understanding of mental illness; they may be unable to correctly recognize mental problems or may have incorrect beliefs about the effectiveness of treatment interventions, and thus become more reluctant to seek help from professionals. It could be that instead of social support and self-reliance, help-seeking patterns of the samples are more influenced by their mental health literacy. The samples in the study are Filipinos and according to Baello and Mori (2007), only a limited number of Filipinos are mental health literate. Filipinos may have not yet fully realized the value of mental health in the same way as they realized the importance of physical health. To note, according to Tan and Tan (2008), the notions of sickness in Philippine folklore 
have not yet emphasized the differences between physical and mental illness. Severe mental health illnesses, for instance, are either perceived as a person's responsibility or as an individual's fragile characteristic

(Thompson, Manderson, Woelz-Stirling, Cahill, \& Kelaher, 2002), with this limited knowledge, knowing how to identify when the difficulties they are facing are beyond the normal threshold is a struggle. One study reported that young people were aware of their distress, but continuously altered their definition of what was "normal" distress to avoid seeking help (Eisenberg et al., 2007).

Another possible explanation could be a stigma that persists in the culture of Filipinos. Some Filipinos are still suffering from stigmatizing attitudes and discriminatory behaviors (Tuliao, 2014). Rivera and Antonio (2017) stated that demeaning terms such as "abnoy" and "baliw" which both translate as crazy, are even incorporated in casual conversations among Filipinos. The labeling of oneself as socially unacceptable for having psychological problems could influence an individual's choice to ask for help (Vogel \& Wester, 2003; Corrigan \& Watson, 2002). As in a sample of undergraduate students, Cheng, McDermott, and Lopez (2015) found that more prominent levels of self-stigma predicted weaker intentions to access counseling services.

Also, prominent among Filipinos was a general concern about what others, including the source of help, might think of them if they were to seek help. This could be seen as "hiya" in the culture of Filipinos. It is characterized as an excruciating feeling that occurs from either real or perceived negative evaluation of society (Bulatao, 1964). Therefore, if having psychological problems is disliked or frowned upon then people will do everything to keep it hidden from the world.

Aside from the factors mentioned above, religiosity might also play a role in individuals' help-seeking patterns. Countries like the Philippines have a powerful adherence to God (Braun \& Browne, 1998) and these religious values strengthen customary respect for relying on God. One possible reason why results also did not reinforce the importance of self-reliance in the treatmentseeking process could be because instead of self-reliance, reliance on God is more associated with seeking or not seeking professional help for the samples in the study. They usually resort to prayers first and unfortunately; no other steps follow such as seeking professional help as they tend to think that it is just another problem that can be overcome because their faith won't fail them. Literature also suggests that a high level of spirituality was related with lower mental health helpseeking and that individuals tend to leave most of their problems even mental health problems to God (Hermannsdóttir, \& Ægisdóttir, 2016; Bradley, Schwartz, \& Kaslow, 2005; Abe-Kim, Gong, \& Takeuchi, 2004).

Furthermore, regardless of the level of self-reliance and social support, if a person does not have the financial capability to seek professional help, he/she won't seek help. Thus, it may also be important to look at the possible role played by an individual's financial support. There are some countries, for instance, the Philippines, where the most pressing issue is poverty, and as such, financial difficulty is a factor. As Chen et al., (2013) suggest, financial difficulty keeps individuals from obtaining mental health services. Also, according to the TPB, individuals are likely to do something when they have the means to do it, and when they believe that such behavior will result in valuable outcomes. It could be that while a person may have a positive attitude toward helpseeking, he/she does not have a sufficient degree of actual control over the behavior. This degree of control over the behavior may also include the perception of financial control. For example, even if the individual perceives professional help-seeking as pleasant, when he/she does not have the financial means to access this help, he/she will not seek help. 


\section{Only Social Support Predicts Actual Help-Seeking behavior through Help-Seeking Intentions and also, it has an additive effect on the relationship among Help-Seeking Attitudes, Intentions, and Behavior}

Similar to many previous studies, social support was observed to be predictive of help-seeking intentions. More so, the findings of the study revealed that social support predicts actual helpseeking behavior through help-seeking intentions. These initial findings were also confirmed in the post hoc analysis using simple mediation. The present study also uncovered that adding social support in the model could increase help-seeking attitudes and therefore intensify help-seeking intentions. When one has positive attitudes toward help-seeking and high social support, one could expect a surge in the intentions to seek help. This denotes a reliance-on-others-promoting effect of social support which consequently increases intentions and behaviors. Correspondingly, while many studies propose a negative relationship, with social support inhibiting the use of mental health services (Vogel et al., 2005), the current study recommends the opposite. To the knowledge of the researcher, this is a novel result as previous studies argued that having high perceived social support from significant others could discourage one from planning to seek professional psychological help (Turner \& Quinn, 1999; Lukito Setiawan, 2006).

The present study found that having high social support can increase intentions. This suggests that even in the presence of supportive social networks, these individuals will still more likely intend to seek formal services for mental healthcare. Taking into consideration the theory of planned behavior may allow one to understand the findings in the study. According to the theory, the intention is the immediate antecedent of behavior and is itself a function of attitude toward the behavior, subjective norm, and perceived behavioral control. In TBP, the subjective norm is a function of normative beliefs, which represent perceptions of specific salient others' preferences about whether one should or should not engage in a behavior. Social support which is defined in the study as a person's perceived social support that comes from one's social network could be a part of this variable which also explains why it can predict help-seeking intention. According to the TPB, individuals are more likely to engage in a behavior that they believe will result in valuable outcomes and in which their significant others approve of the action they intend to do. Thus, an individual with positive attitudes about seeking professional help, and who perceives social support for these behaviors from key referent others will likely seek professional help. More so, when the individual's social support approves and sees professional help-seeking as positive, he/she may likewise develop the same feeling because his/her referent groups do.

Another possible explanation could be the value of dependence within the Filipino family. Social support promoting reliance on others could be better understood in a Filipino context. For instance, according to Warner (2003), Filipinos have a social value of "pagsangguni", or the seeking of opinions before making a decision. For Filipinos, decisions are made only after a mutual agreement among the closest social networks. Hence, suggesting that social support among Filipinos may have originated from this social value. And as such, when this is high, they can be more prompted to finally decide to seek professional help.

\section{Conclusion}

Mental health help-seeking is a multifaceted experience that varies depending on a wide range of individual, interpersonal, and sociocultural factors. Research should continue to examine the path from help-seeking attitudes to the willingness to seek help to actual help-seeking and explore factors hypothesized above that prevent or influence individuals from seeking actual help from formal sources across cultures.

Several limitations of the present study should be considered when going through the results and when planning for future research. First, a potential selection bias within the use of the 
snowball sampling method, as well as the use of an internet survey mostly for gathering data could have excluded people that might have possibly changed the results. A further limitation was that one of the variables was assessed via a one-item or dichotomous ( $\mathrm{Yes} / \mathrm{No}$ ) measure. Furthermore, as specified over, one should consider using more nuanced, multi-item instruments to investigate relationships more comprehensively and to accurately capture the diverse experiences of individuals with mental health concerns. The instrument used to measure self-reliance did not yield strong internal reliability which could also have affected the results, thus suggesting future researchers utilize a more reliable scale when measuring self-reliance. Another drawback is the way that other potentially-moderating factors of the association among attitudes toward help-seeking, intentions, and actual help-seeking behavior were not measured, such as self-stigma, religiosity, socio-demographic factors, or even identification with a culture or subculture with solid convictions. For instance, future research should assess the notion of financial support in the helpseeking process specifically when research would involve respondents from and developing countries such as the Philippines as a few people may choose not to look for professional health services in light of monetary reasons. Adding the role of financial control would help improve the predictive ability of the model. Also, it may be imperative to examine a group of cultural values/traits to determine how they may be related to expert mental health help-seeking behavior. An extensive and in-depth examination of how cultural norms differentially affect help-seeking behaviors in mental health settings will assist researchers and practitioners in identifying normative factors that promote or impede the pathway to help-seeking for mental health problems.

Nevertheless, the study likewise had a few qualities, most remarkably a large sample size, robust statistical techniques, and a crucial topic that was moderately unexplored in the previous literature on emerging adults' help-seeking patterns. The sample of this study was a snowball, yet, the heterogeneity and also the sample size are thought to be strengths for the objective of the analyses. The study was also able to represent gender in equal balance. The findings of this study provided research-based data in light of how to investigate a potential moderated mediation effect of a certain variable in an existing relationship. More importantly, from the knowledge of the researcher, this study is among the first to capture and investigate the relationship among all the three major elements of help-seeking pattern, namely attitudes, intentions, and help-seeking, where previous studies focused only on one or two. The study was also able to confirm and support the most recent proposed model of help-seeking by Rickwood \& Thomas (2012).

Furthermore, these findings have provided necessary implications for interventions in mental health help-seeking. Ultimately, the discrepancies of results of the study from previous research could also enlighten clinicians about considering variations in help-seeking patterns among people across cultures. Mainly, health professionals and researchers should be informed about making false speculations that do not seem to be supported by particular research findings in a particular culture. Recommendations and suggestions for interventions should comprise a culturally sensitive, comprehensive, and holistic approach that tends to the individual and the cultural milieu.

From a clinical perspective, it may be useful to note that cultural differences are likely to also impact the adherence to and outcomes of mental health care in treatment planning. If it is the case that cultural factors play a comparable (or perhaps even more important) role in predicting the use of mental health services, either initial contact or adherence to follow-up appointments, this information has significant implications for clinical treatment and interventions. Also, public health intervention and outreach efforts aimed at identifying those with an unmet need for treatment may benefit from this information. For instance, intake screening procedures that include cultural values/traits may be of prognostic significance for predicting adherence or risk of treatment discontinuation. 
Also, given the limited awareness on mental health among Filipinos, it may be important to consider the value of a national health education campaign to increase the public's understanding of certain mental health problems and hence reduce unwanted variation in the use of mental health services. Facilitating a sense of familiarity with and awareness of service options amongst individuals might help to bridge the gap between people's needs and utilization of mental health services. In particular, peoples' stigma-related characteristics could constitute a key target for such efforts, as their intended stigmatizing behaviors bear a great impact on help-seeking. Public health intervention efforts aimed at improving access to care may benefit from including these issues in the planning of outreach and community-based programs. Overall, these findings suggest that the inclusion of cultural influences in upcoming efforts to identify factors that contribute to the relationship between mental disorders and future mental health may be worthwhile.

The findings also underscore the significance of social support in affecting mental health help-seeking behaviors. The present findings are encouraging because social support may serve as an important means of overcoming other barriers associated with mental health treatment. Being able to rely on and disclose to family, friends and significant other may also normalize the act of seeking psychological help. It is clear that while there may be various social influences on the helpseeking process, the family will still top the list. As such, interventions should also be targeted at this group of people. These people may also need help to develop mental health literacy so that they would know when to access professional help, where to obtain such help and how to encourage their child to accept such aid. Therefore, strengthening social support that has an accurate knowledge of mental health can be an intervention on the part of clinicians to aid helpseeking behavior.

\section{References}

Abdulla, T., \& Brown, T. L. (2011). Mental illness stigma and ethnocultural beliefs, values, and norms: An integrative review. Clinical psychology review, 31(6), 934-948.

Abe-Kim, J., Gong, F., \& Takeuchi, D. (2004). Religiosity, spirituality, and help-seeking among Filipino Americans: Religious clergy or mental health professionals? Journal of Community Psychology, 32(6), 675-689.

Ægisdóttir, S., \& Gerstein, L. H. (2009). Beliefs About Psychological Services (BAPS): development and psychometric properties. Counselling Psychology Quarterly, 22(2), 197-219.

Ægisdóttir, S., O'Heron, M., Hartong, J., Haynes, S., \& Linville, M. (2011). Enhancing attitudes and reducing fears about mental health counseling: An analog study. Journal of Mental Health Counseling, 33(4), 327-346.

Ajzen, I., \& Fishbein, M. (2000). Attitudes and the attitude-behavior relation: Reasoned and automatic processes. European review of social psychology, 11(1), 1-33.

American Psychiatric Association. (2013). Diagnostic and statistical manual of mental disorders (DSM-5®). American Psychiatric Pub.

Armitage, C. J., \& Conner, M. (2001). Efficacy of the theory of planned behavior: A meta-analytic review. British journal of social psychology, 40(4), 471-499.

Azjen, I. (1991). The theory of planned behavior. Organizational behavior and buman decision processes, 50(2), 179-211.

Baello, J., \& Mori, L. (2007). Asian values adherence and psychological help-seeking attitudes of Filipino Americans. Journal of Multicultural, Gender and Minority Studies, 1(1), 1-14.

Bagley, K. D. (2011). Cross-racial comparison of cognitive factors influencing help-seeking (Doctoral dissertation, George Mason University).

Bradley, R., Schwartz, A. C., \& Kaslow, N. J. (2005). Posttraumatic stress disorder symptoms among lowincome, African American women with a history of intimate partner violence and suicidal 
behaviors: Self-esteem, social support, and religious coping. Journal of Traumatic Stress: Official Publication of The International Society for Traumatic Stress Studies, 18(6), 685-696.

Braun, K. L., \& Browne, C. V. (1998). Perceptions of dementia, caregiving, and help-seeking among Asian and Pacific Islander Americans. Health \& Social Work, 23(4), 262-274.

Britt, T. W., Bennett, E. A., Crabtree, M., Haugh, C., Oliver, K., McFadden, A., \& Pury, C. L. (2011). The theory of planned behavior and reserve component veteran treatment seeking. Military Psychology, 23(1), 82.

Brown, G. W., \& Harris, T. O. (Eds.). (1989). Life events and illness. Guilford Press.

Bujang, M. A., Sa'at, N., \& Bakar, T. M. I. T. A. (2017). Determination of minimum sample size requirement for multiple linear regression and analysis of covariance-based on experimental and nonexperimental studies. Epidemiology, Biostatistics, and Public Health, 14(3).

Bulatao, J. C. (1964). Hiya. Philippine Studies, 12(3), 424-438.

Buri, J. (2018). Negative life events scale for students (NLESS). College Student Journal, 52(3), 361-367.

Cairns, S. L., Massfeller, H. F., \& Deeth, S. C. (2010). Why do postsecondary students seek counseling? Canadian Journal of Counselling and Psychotherapy (Online), 44(1), 34.

Carlton, P. A., \& Deane, F. P. (2000). Impact of attitudes and suicidal ideation on adolescents' intentions to seek professional psychological help. Journal of Adolescence, 23(1), 34-45.

Chen, L. Y., Crum, R. M., Martins, S. S., Kaufmann, C. N., Strain, E. C., \& Mojtabai, R. (2013). Service use and barriers to mental health care among adults with major depression and comorbid substance dependence. Psychiatric Services, 64(9), 863-870.

Cheng, H. L., McDermott, R. C., \& Lopez, F. G. (2015). Mental health, self-stigma, and help-seeking intentions among emerging adults: An attachment perspective. The Counseling Psychologist, 43(3), 463487.

Choi, J. Y. (2008). Seeking health care: Marshallese migrants in Hawai 'i. Ethnicity and Health, 13(1), 73-92.

Chong, S. A., Abdin, E., Sherbourne, C., Vaingankar, J., Heng, D., Yap, M., \& Subramaniam, M. (2012). The treatment gap in common mental disorders: the Singapore perspective. Epidemiology and Psychiatric Sciences, 21(2), 195-202.

Christopher, M. S., Skillman, G. D., Kirkhart, M. W., \& D'Souza, J. B. (2006). The effect of normative and behavioral persuasion on help-seeking in Thai and American college students. Journal of Multicultural Counseling and Development, 34(2), 80-93.

Corrigan, P. W., \& Watson, A. C. (2002). The paradox of self-stigma and mental illness. Clinical Psychology: Science and Practice, 9(1), 35-53.

Eisenberg, D., Golberstein, E., \& Gollust, S. E. (2007). Help-seeking and access to mental health care in a university student population. Medical care, 45(7), 594-601.

Elliot, A. J., \& Covington, M. V. (2001). Approach and avoidance motivation. Educational Psychology Review, 13(2), 73-92.

Franz, A. (2012). Predictors of help-seeking behavior in emerging adults.

Grant, J. E., \& Potenza, M. N. (2010). Young Adult Mental Health: Oxford University Press.

Gulliver, A., Griffiths, K. M., \& Christensen, H. (2010). Perceived barriers and facilitators to mental health help-seeking in young people: a systematic review. BMC psychiatry, 10(1), 113.

Hammer, J. H., \& Spiker, D. A. (2018). Dimensionality, reliability, and predictive evidence of validity for three help-seeking intention instruments: ISCI, GHSQ, and MHSIS. Journal of counseling psychology, 65(3), 394.

Hammer, J. H., Parent, M. C., \& Spiker, D. A. (2018). Mental Help-Seeking Attitudes Scale (MHSAS): Development, reliability, validity, and comparison with the ATSPPH-SF and IASMHS-PO. Journal of counseling psychology, 65(1), 74.

Harewood, J. E. (2009). Social and personal determinants of help-seeking intentions among Black college students. State University of New York at Albany.

Hayes, A. F. (2015). An index and test of linear moderated mediation. Multivariate Behavioral Research, 50(1), $1-22$. 
Hermannsdóttir, B. S., \& Aegisdottir, S. (2016). Spirituality, Connectedness, and Beliefs About Psychological Services Among Filipino Immigrants in Iceland. The Counseling Psychologist, 44(4), 546572.

Jordanova, V., Stewart, R., Goldberg, D., Bebbington, P.E., Brugha, T., Singleton, N., Lindesay, J.E., Jenkins, R., Prince, M. and Meltzer, H., 2007. Age variation in life events and their relationship with common mental disorders in a national survey population. Social Psychiatry and Psychiatric Epidemiology, 42(8), 611-616.

Klandermans, B. (2013). Survey Research. The Wiley-Blackwell Encyclopedia of Social and Political Movements.

Labouliere, C. D., Kleinman, M., \& Gould, M. S. (2015). When self-reliance is not safe: associations between reduced help-seeking and subsequent mental health symptoms in suicidal adolescents. International journal of environmental research and public health, 12(4), 3741-3755.

Lukito Setiawan, J. (2006). Willingness to seek counseling and factors that facilitate and inhibit the seeking of counseling in Indonesian undergraduate students. British Journal of Guidance \& Counselling, 34(3), 403-419.

Mackenzie, C. S., Gekoski, W. L., \& Knox, V. J. (2006). Age, gender, and the underutilization of mental health services: the influence of help-seeking attitudes. Aging and Mental Health, 10(6), 574-582.

Martínez-Hernáez, A., DiGiacomo, S. M., Carceller-Maicas, N., Correa-Urquiza, M., \& Martorell-Poveda, M. A. (2014). Non-professional-help-seeking among young people with depression: a qualitative study. BMC psychiatry, 14(1), 1-11.

Mo, P. K., \& Mak, W. W. (2009). Help-seeking for mental health problems among Chinese. Social psychiatry and psychiatric epidemiology, 44(8), 675-684.

Nagai, S. (2015). Predictors of help-seeking behavior: Distinction between help-seeking intentions and help-seeking behavior. Japanese Psychological Research, 57(4), 313-322.

National Collaborating Centre for Mental Health. (2004). Depression: Management of Depression in Primary and Secondary Care. London: National Institute for Clinical Excellence.

Perlick, D. A., Hofstein, Y., \& Michael, L. A. (2010). Barriers to mental health service use in young adulthood. Young adult mental health, 195-205.

Powell, G. N., \& Kotschessa, B. (1995). Factors that influence professional employees' willingness to seek counseling. Psychological Reports, 77(3), 872-874.

Reavley, N. J., Yap, M. B., Wright, A., \& Jorm, A. F. (2011). Actions taken by young people to deal with mental disorders: findings from an Australian national survey of youth. Early intervention in psychiatry, 5(4), 335-342.

Rickwood, D. J., Deane, F. P., \& Wilson, C. J. (2007). When and how do young people seek professional help for mental health problems? Medical Journal of Australia, 187(7), S35-S39.

Rickwood, D., \& Thomas, K. (2012). Conceptual measurement framework for help-seeking for mental health problems. Psychology Research and Behavior Management, 5, 173.

Rickwood, D., Deane, F. P., Wilson, C. J., \& Ciarrochi, J. (2005). Young people's help-seeking for mental health problems. Australian e-journal for the Advancement of Mental health, 4(3), 218-251.

Rivera, A. K. B., \& Antonio, C. A. T. (2017). Mental health stigma among Filipinos: Time for a paradigm shift. Philippine Journal of Health Research and Development, 21(2), 20-24.

Rubio, C. T., \& Lubin, B. (1986). College student mental health: A person-environment interactional analysis. Journal of clinical psychology, 42(1), 205-212.

Salaheddin, K., \& Mason, B. (2016). Identifying barriers to mental health help-seeking among young adults in the UK: a cross-sectional survey. British Journal of General Practice, 66(651), e686-e692.

Schomerus, G., Matschinger, H., \& Angermeyer, M. C. (2009). Attitudes that determine willingness to seek psychiatric help for depression: a representative population survey applying the Theory of Planned Behaviour. Psychological Medicine, 39(11), 1855-1865.

Segal, D. L., Coolidge, F. L., Mincic, M. S., \& O'riley, A. (2005). Beliefs about mental illness and willingness to seek help: A cross-sectional study. Aging \& Mental Health, 9(4), 363-367.

Solomon, Z., Mikulincer, M., \& Flum, H. (1989). The implications of life events and social integration in the course of combat-related post-traumatic stress disorder. Social Psychiatry and Psychiatric Epidemiology, 24(1), 41-48. 
Tan, M. L., \& Tan, M. T. (2008). Revisiting usog, pasma, kulam. UP Press.

Tanner, J. L. (2016). Mental health in emerging adulthood.

ten Have, M., de Graaf, R., Ormel, J., Vilagut, G., Kovess, V., Alonso, J., \& ESEMeD/MHEDEA 2000 Investigators. (2010). Are attitudes towards mental health help-seeking associated with service use? Results from the European Study of Epidemiology of Mental Disorders. Social psychiatry and psychiatric epidemiology, 45(2), 153-163.

Thompson, S., Manderson, L., Woelz-Stirling, N., Cahill, A., \& Kelaher, M. (2002). The social and cultural context of the mental health of Filipinas in Queensland. Australian and New Zealand Journal of Psychiatry, 36(5), 681-687.

Topkaya, N. (2014). Gender, Self-Stigma, and Public Stigma in Predicting Attitudes toward Psychological Help-Seeking. Educational Sciences: Theory and Practice, 14(2), 480-487.

Tuliao, A. P. (2014). Mental health help-seeking among Filipinos: a review of the literature. Asia Pacific Journal of Counselling and Psychotherapy, 5(2), 124-136.

Turner, A. L., \& Quinn, K. F. (1999). College students' perceptions of the value of psychological services: A comparison with APA's public education research. Professional Psychology: Research and Practice, 30(4), 368.

Vogel, D. L., \& Wester, S. R. (2003). To seek help or not to seek help: The risks of self-disclosure. Journal of Counseling Psychology, 50(3), 351.

Vogel, D. L., Wester, S. R., Wei, M., \& Boysen, G. A. (2005). The Role of Outcome Expectations and Attitudes on Decisions to Seek Professional Help. Journal of Counseling Psychology, 52(4), 459.

Warner, M. (2003). Culture and Management in Asia. Psychology Press.

Yusuke, U. (2017). Correlations between formal help-seeking behavior for psychological problems and help-seeking intentions and attitudes. The Japanese Journal of Psychology, 88(2), 191-196.

Zartaloudi, A. E. (2010). Help-seeking as a threat to self-reliance and self-esteem of an individual with mental health problems: a questionnaire survey. The Scientific Journal of the Hellenic Regulatory Body of Nurses, 3(3), 67-74.

Zimet, G. D., Dahlem, N. W., Zimet, S. G., \& Farley, G. K. (1988). The multidimensional scale of perceived social support. Journal of personality assessment, 52(1), 30-41.

Zivin, K., Eisenberg, D., Gollust, S. E., \& Golberstein, E. (2009). Persistence of mental health problems and needs in a college student population. Journal of affective disorders, 117(3), 180-185. 


\section{Appendix}

\section{Survey Questionnaires}

\section{NEGATIVE LIFE EVENTS SCALE FOR STUDENTS (NLESS)}

"Listed below are several life events that are often stressful for people who experience them. For each of the following events, please indicate whether or not you have experienced that event in the past six months by placing a check mark $(\boldsymbol{V})$ in the space before the item.

1. Death of a close family member (e.g., mother, father, sister, brother)

2. Death of your best friend (or a very close friend)

3. Serious illness / injury to a close family member (e.g., mother, father, sister, brother)

4. Serious personal illness / injury to yourself

5 . Serious illness / injury to your best friend (or a very close friend)

6. Your parents going through a separation/divorce.

7. Having a family get arrested and/or ending up in jail.

8. Having been arrested and/or placed in jail yourself.

9. You, having been victim of an assault, robbery or some other crime.

10. Having a parent who has been laid off/fired or who has been out of work for an

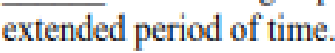

11. You're having a serious break-up with boyfriend / girlfriend.

12. Your family experiencing a major financial pressures.

13. You're personally experiencing a major and/or chronic financial strain.

14. Having a parent or sibling who is struggling with addiction or some other psychological / emotional problem. problem.

15. You're personally coping with addiction or some other psychological / emotional

16. You, having been cheated on by boyfriend / girlfriend.

17. You, having failed several courses and/or been placed on academic probation or expelled from school.

other.

18. Your parents having serious ongoing conflicts/disagreements/arguments with each

19. You're experiencing ongoing conflict/disagreements/arguments with your parents and/or other family members.

20. You're experiencing abuse / violence at home.

21. Your family losing house through fire, flood, foreclosure or other disaster/

22. You're experiencing unwanted sexual behaviors imposed on you by another person against your will.

23. You're experiencing an unwanted pregnancy (either yourself or you, being the father)

24. You're experiencing ongoing conflict/disagreements/arguments with your best friend/s

25 . You're having a trouble with your boss (e.g., your boss is consistently critical of you,

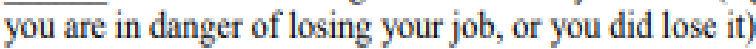




\section{MENTAL HELP SEEKING ATTITUDES SCALE (MHSAS)}

INSTRUCTIONS: For the purposes of this survey, "mental health professionals" include psychologists, psychiatrists, clinical social workers, and counselors. Likewise, "mental health concerns" include issues ranging from personal difficulties (e.g., loss of a loved one) to mental illness (e.g., anxiety, depression).

Please mark the circle that best represents your opinion. For example, if you feel that your seeking help would be extremely useless, you would mark the circle closest to "useless." If you are undecided, you would mark the " 0 " circle. If you feel that your seeking help would be slightly useful, you would mark the "1" circle that is closer to "useful."

If I had a mental health concern, seeking help from a mental health professional would be...

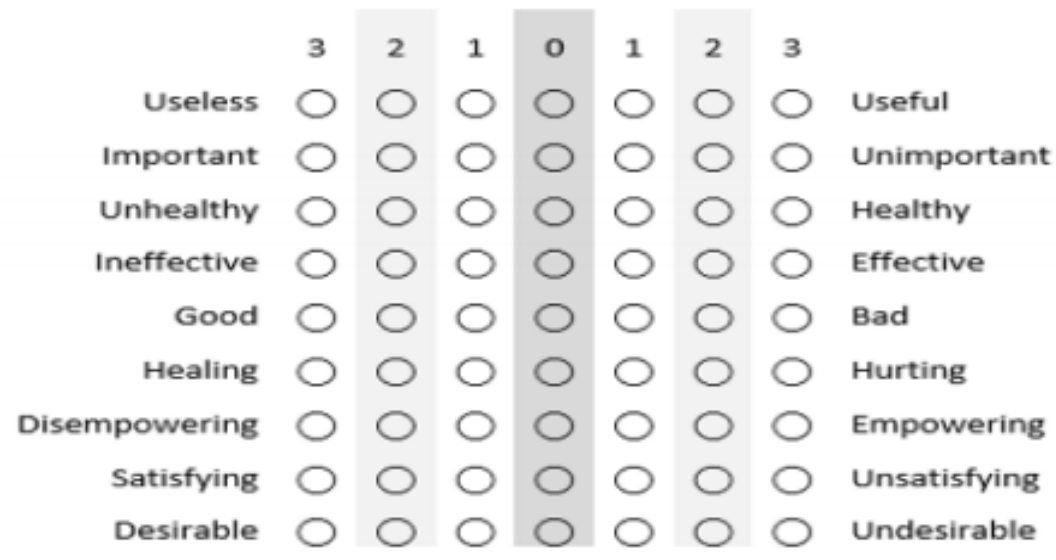

\section{MENTAL HELP SEEKING INTENTION SCALE (MHSIS)}

INSTRUCTIONS: For the purposes of this survey, "mental health professionals" include psychologists, psychiatrists, clinical social workers, and counselors. Likewise, "mental health concerns" include issues ranging from personal difficulties (e.g., loss of a loved one) to mental illness (e.g., anxiety, depression). Please mark the box that best represents your opinion.

If I had a mental health concern, I would intend to seek help from a mental health professional.

\begin{tabular}{|c|l|l|l|l|l|c|}
\hline $\begin{array}{c}1 \\
\begin{array}{c}\text { Extremely } \\
\text { unlikely) }\end{array}\end{array}$ & 2 & 3 & 4 & 5 & 6 & $\begin{array}{c}7 \\
\text { (Extremely } \\
\text { likely) }\end{array}$ \\
\hline
\end{tabular}

If I had a mental health concern, I would try to seek help from a mental health professional.

\begin{tabular}{|c|c|c|c|c|c|c|}
\hline $\begin{array}{c}1 \\
\begin{array}{c}\text { Extremely } \\
\text { unlikely) }\end{array}\end{array}$ & 2 & 3 & 4 & 5 & 6 & $\begin{array}{c}7 \\
\text { (Extremely } \\
\text { likely) }\end{array}$ \\
\hline
\end{tabular}

If I had a mental health concern, I would plan to seek help from a mental health professional.

\begin{tabular}{|c|l|l|l|l|l|c|}
\hline $\begin{array}{c}1 \\
\begin{array}{c}\text { Extremely } \\
\text { unlikely) }\end{array}\end{array}$ & 2 & 3 & 4 & 5 & 6 & $\begin{array}{c}7 \\
\text { (Extremely } \\
\text { likely) }\end{array}$ \\
\hline
\end{tabular}




\section{ACTUAL HELP-SEEKING BEHAVIOR}

Within the past six months, have you sought help from a professional for mental health concerns which include but are not limited to problems coping with distressing situations or life event, anxiety, and or depression? YES NO

\section{SELF-RELIANCE FOR COPING WITH MENTAL HEALTH PROBLEMS}

INSTRUCTIONS: The following questions refer to your views of getting mental health treatment (e.g. counseling, medication) were you to develop a stress or emotional problem, as well as your views of mental health professionals (e.g. psychologists, counselors, psychiatrists). Using the scale provided, rate your extent of agreement with the following statements:

\begin{tabular}{|c|c|c|c|c|c|}
\hline Statements & $\begin{array}{l}\text { Strongly } \\
\text { Disagree } \\
\text { (1) }\end{array}$ & $\begin{array}{l}\text { Disagree } \\
\text { (2) }\end{array}$ & $\begin{array}{l}\text { Neither agree } \\
\text { Nor Agree } \\
\text { (3) }\end{array}$ & \begin{tabular}{|l|} 
Agree \\
$(4)$
\end{tabular} & \begin{tabular}{|l} 
Strongly \\
Agree \\
$(5)$ \\
\end{tabular} \\
\hline $\begin{array}{l}\text { 1. I prefer to handle problems } \\
\text { myself as opposed to seek } \\
\text { mental health treatment. }\end{array}$ & & & & & \\
\hline $\begin{array}{l}\text { 2. Strong people can get over } \\
\text { psychological problems by } \\
\text { themselves. }\end{array}$ & & & & & \\
\hline $\begin{array}{l}\text { 3. Psychological problems tend } \\
\text { to work themselves out } \\
\text { without heln }\end{array}$ & & & & & \\
\hline
\end{tabular}

\section{MULTIDIMENSIONAL SCALE OF PERCEIVED SOCIAL SUPPORT}

Instructions: We are interested in how you feel about the following statements. Read each statement carefully. Indicate how you feel about each statement.

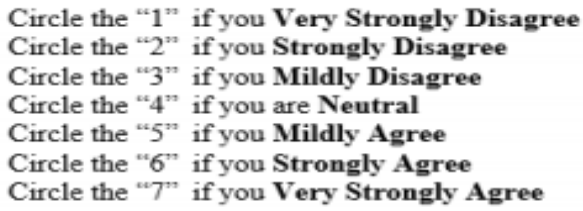

1. There is a special person who is around when I am in need.

2. There is a special person with whom I can share $\quad \begin{array}{lllllllllllllll}1 & 2 & 3 & 4 & 5 & 6 & 7 & \text { SO }\end{array}$ my joys and sorrows.

3. My family really tries to help me. $\quad \begin{array}{lllllllllll} & 1 & 2 & 3 & 4 & 5 & 6 & 7 & \text { Fam }\end{array}$

4. I get the emotional help and support I need from $110 \begin{array}{lllllllll} & 1 & 3 & 4 & 5 & 6 & 7 & \text { Fam }\end{array}$ my family.

5. I have a special person who is a real source of $\quad \begin{array}{llllllllllllll} & 1 & 2 & 3 & 4 & 5 & 6 & 7 & \text { SO }\end{array}$ comfort to me

6. My friends really try to help me:

$\begin{array}{lllllllllll}\text { I can count on my friends when things go wrong. } & 1 & 2 & 3 & 4 & 5 & 6 & 7 & \text { Fri }\end{array}$

I can talk about my problems with my family. $\quad \begin{array}{llllllllll} & 1 & 2 & 3 & 4 & 5 & 6 & 7 & \text { Fam }\end{array}$

$\begin{array}{lllllllllll}\text { I have friends with whom I can share my joys } & 1 & 2 & 3 & 4 & 5 & 6 & 7 & \text { Fri }\end{array}$ and sorrows.

10. There is a special person in my life who cares $\quad \begin{array}{llllllllllll} & 1 & 2 & 3 & 4 & 5 & 6 & 7 & \text { SO }\end{array}$ about my feelings.

11. My family is willing to help me make decisions. $\begin{array}{llllllllll}1 & 2 & 3 & 4 & 5 & 6 & 7 & \text { Fam }\end{array}$

12. I can talk about my problems with my friends. $\quad \begin{array}{lllllllllll}1 & 2 & 3 & 4 & 5 & 6 & 7 & \text { Fri }\end{array}$

The items tended to divide into factor groups relating to the source of the social support, namely family (Fam), friends (Fri) or significant other (SO). 